\title{
FACTORS INFLUENCING KNOWLEDGE TRANSFER FROM FACULTY TO PHD STUDENTS
}

\author{
Slobodan M. Janković, Dejan Z. Aleksić, \\ Andriana M. Bukonjić, Dusan Lj. Tomović
}

Faculty of Medical Sciences, University of Kragujevac

ФАКТОРИ КОЈИ УТИЧУ НА ПРЕНОС ЗНАњА СА
УНИВЕРЗИТЕТСКИХ НАСТАВНИКА
НА СТУДЕНТЕ ДОКТОРСКИХ СТУДИЈА

Слободан М. Јанковић, Дејан 3. Алексић, Андриана М. Букоњић, Душан Љ. Томовић

Факултет медицинских наука, Универзитет у Крагујевцу

\begin{abstract}
A phenomenon that does not quite rise to the level of research misconduct occurs when faculty responsible for assisting $\mathrm{PhD}$ students fail to fulfill that role. The factors that may affect readiness of faculty to help $\mathrm{PhD}$ students with a methodological issue concerning sampling that was essential for designing a dissertation were revealed in this study - a simulated student-professor interaction study. Of 153faculty asked to assist, $47 \%$ failed to respond and of those who responded, only $21 \%$ made an appointment to help the student. Results of our study showed that responsible behavior of university professors in regard to helping $\mathrm{PhD}$ students was more likely if the faculty member was younger, had more recently been employed and had higher publication and citation scores.
\end{abstract}

Key words: University teacher, $\mathrm{PhD}$ student, scientific irresponsibility

\section{САЖЕТАК}

Појава која се не може назвати неетичним понашањем у образовању али представља значајан проблем настаје када наставници задужени за одржавање наставе на докторским студијама не пружају стручну помоћ када им студенти то затраже. У овој студији, која се бави симулацијом интеракције студената докторских студија са наставницима, испитани су фактори који могу утицати на спремност наставника да студентима разјасне методолошка питања у вези са дизајном њихове будуће докторске тезе. Од 153 наставника којима је тражена помоћ везана за припрему дизајна тезе $47 \%$ није уопште одговорило, а од оних који су одговорили само $21 \%$ је заказало састанак са студентима. Наша студија је показала да су универзитетски наставници спремнији да помогну студентима докторских студија уколико су млађи, скорије почели да раде на универзитету и ако имају више публикација и већи индекс цитираности.

\footnotetext{
Контакт: Prof. Slobodan M. Jankovic, MD, DSc, MSc, Prim.

Faculty of Medical Sciences, SvetozaraMarkovica Street, 69, 34000 Kragujevac, Serbia e-mail: slobodan.jankovic@medf.kg.ac.rs
} 
Кључне речи: универзитетски наставници, студент докторских студија, неодговорно понашање у образовању

\section{INTRODUCTION}

Knowledge transfer from faculty to $\mathrm{PhD}$ students is vital to both the process of education and to the quality of the dissertations that are produced. Readiness to make open, sincere and complete transfer of necessary knowledge is an ethical responsibility of faculty ${ }^{1}$. Is the failure of faculty to fulfill this role a form of scientific misconduct? While all scientific societies and professions consider this a duty of faculty, the passive failure to assist $\mathrm{PhD}$ students does not rise to the level of "scientific misconduct." However, many of the faculty do not even recognize that passive or active avoidance of open and sincere knowledge transfer is a form of irresponsible and unethical behavior ${ }^{2,3,4,5}$, which could partly explain ubiquitous nature of this phenomenon ${ }^{3}$.

In everyday work, young researchers are exposed to both positive and negative models of behavior in the research community ${ }^{6}$.Those who want to help in formulating ideas and designing dissertations of those who are in the beginning of their research carrier are invaluable to science and to their own institutions ${ }^{6,7}$. Such teachers are able to enhance development and productivity of young researchers ${ }^{8}$.

Communication between senior and young researchers is also important for the maintenance and promotion of research traditions ${ }^{1}$. Experienced researchers are rich with practical ideas for research. Their main tasks are to identify potential of young researchers, stimulate their independent research activity and facilitate their integration into the scientific community ${ }^{8,9}$. Although importance of interaction and knowledge transfer between faculty and $\mathrm{PhD}$ students for appropriate development of young researchers is widely recognized in medical literature ${ }^{10,11}$, it is surprising that published research on this topic is so scarce. Although it was observed that younger faculty spare less time interacting with undergraduate students ${ }^{12}$, we are practically unaware what factors may influence willingness of faculty to openly and without reserve share their knowledge about research design with $\mathrm{PhD}$ students and young researchers in general.

The aim of this study was to reveal factors that may affect readiness of faculty to help $\mathrm{PhD}$ students with some methodological issues essential for setting up a dissertation. This problem was easy to study within the Faculty of Medical Sciences, University of Kragujevac, Kragujevac, Serbia, where $\mathrm{PhD}$ students do not have specifically formulated committees, but are expected to find and request help from faculty in their specific area of study, and faculty, in turn, are expected to respond helpfully.

\section{METHODS}

\section{Study Design}

The study was designed as a simulated $\mathrm{PhD}$ student - faculty interaction, where the faculty were not aware that they participated in the study. The intervention consisted of previously prepared request for help with calculation of sample size for the future research which would be included in a $\mathrm{PhD}$ dissertation. Three $\mathrm{PhD}$ students prepared the following letter, and sent it to the faculty of the Faculty of Medical Sciences (FMS), University of Kragujevac, by e-mail, using official e-mail addresses:

\section{„Dear Professor...,}

I am a second year $\mathrm{PhD}$ student and I need some professional help regarding the design of my future $\mathrm{PhD}$ dissertation. In fact, it is the planning of the sample for the dissertation, which is of the case-control study type. After reviewing the list of your publications on the web site of the Faculty of Medical Sciences, I saw that you have a lot of experience in the design of the studies, so please spare some time for me and schedule a meeting in which you will help me to plan the sample size.

\section{Grateful student..."}

Each of the $\mathrm{PhD}$ students sent the letter in weekly aliquots of 10 e-mails during five weeks in 2013/14 school year. In this way, every member of the FMS' faculty received the letter. If a faculty responded to the letter, the students would start correspondence and then went to a meeting, provided that one was appointed by the faculty. All e-mails exchanged with the faculty have been stored in a database for further analysis.

The study was approved by the Ethics Committee of Faculty of Medical Sciences, University of Kragujevac.

\section{Study Population}

Study populationconsisted of all 153 faculty members (assistant professors, associate professors and full professors) employed at the Faculty 
of Medical Sciences, University of Kragujevac, during 2013/14 school year. PhD study program at FMS started in 2005, and was organized according to national legislation, which does not request foundation of specifically formulated committees which would help to $\mathrm{PhD}$ students with setting up of their dissertations. There is only one central committee at FMS which approves or disapproves the proposals of dissertations, and in regard to actual design of their dissertations the students depend on the help from faculty in their specific area of study. Only four years ago for each of the $\mathrm{PhD}$ students was appointed a tutor, but his/her official responsibility is limited to reporting about activities and progress of a student.

\section{Study Variables}

The (dependent variables were the faculty response to the e-mail, time to reply to the email, making appointment, and the type of help (no help, conditioned help, partial help [directing a student to a reference], complete help) to the PhD student.

Possible independent and confounding variables were: gender, research experience of faculty expressed as years of life and duration of employment at university, academic title, research area (preclinical or clinic), teaching surgical or internal diseases subjects, number of publications, number of citations, h-index ${ }^{13}$, number of co-authors, number of citations from2011to2013, and average number of citations per document (according to Scopus ${ }^{14}$ and Web of Knowledge databases ${ }^{15}$ ).

\section{Statistical Analysis}

The data were primarily statistically described using means and standard deviations for continuous variables and percentages for categorical variables. Normality of the data distribution was checked by Kolmogorov-Smirnov test. If normality was confirmed, Student T-test was used to compare the study groups. Otherwise, the nonparametric Mann Whitney U test was used. Categorical variables were compared by Chisquare test. Binary logistic regression was used to identify variables that are associated with the study outcomes. All calculations were performed by statistical software SPSS, version 18 .

\section{Statistical Analysis}

The study populationconsisted of all faculty members employed at FMS: 153 of them, 77 $(51.0 \%)$ female and $75(49.0 \%)$ male. The average age of study participants was $50.01 \pm 7.83$ (SD) years. There were $65(42.5 \%)$ associate professors, 49 (32.0\%) assistant professors, and $39(25.5 \%)$ full professors. The average duration of total employment at university was 16.90 \pm 7.78 (SD) years, and average duration of employment with the current academic title was $4.86 \pm 4.26(\mathrm{SD})$ years.

In total 72 faculties $(47.1 \%)$ did not respond at all to the request for help sent by the e-mail. Sixty-five $(42.5 \%)$ study participants replied to the request after on average $2.42 \pm 3.52$ (SD) days. Six faculties (3.9\%) did not have an available e-mail, while the e-mail addresses of next 10 faculties $(6.5 \%)$ were not functional (an automatic e-mail reply came back to the investigators stating that such e-mail addresses do not exist). Out of the faculties who replied, 32 (20.9\%) of made an appointment with the investigators. Characteristics of the study participants according to their response to the request for knowledge transfer are shown in Table 1.

No help at all was offered by 107 (69.9\%) faculties. They either didn't reply to the e-mail or refused to help by e-mail reply or after meeting students. Partial help (referring to literature or statistical software, or referring to another professional, tutor or mentor) was given by 37 (24.2\%) subjects: seven $(4.6 \%)$ of them also offered mentoring or having insight into the research plan. Only 2 (1.3\%) gave their complete and unconditional support.

In order to understand which characteristics of the faculty contribute to their positive response to the request of the $\mathrm{PhD}$ students, odds ratios for groups which responded to the e-mail, made an appointment and offered help and groups that did not were calculated. Crude odds ratios were compared with odds ratios calculated after adjustment by binary logistic regressions. The results are shown in Tables 2, 3 and 4 . 


\begin{tabular}{|c|c|c|c|c|c|c|c|c|c|c|c|c|}
\hline 7 & $\stackrel{\vec{s}}{\stackrel{s}{\sigma}}$ & 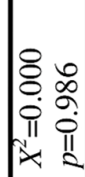 & 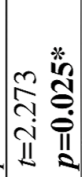 & 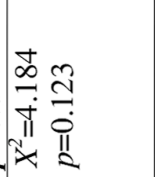 & 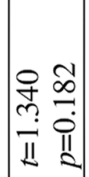 & 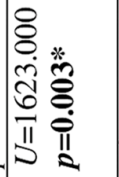 & 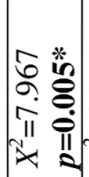 & 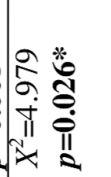 & 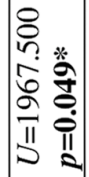 & 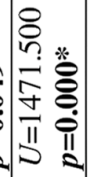 & 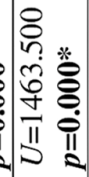 & 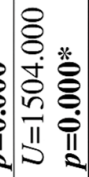 \\
\hline 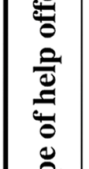 & $\begin{array}{l}\frac{2}{0} \\
\text { 음 } \\
z\end{array}$ & 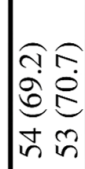 & $\begin{array}{ll}2 & \infty \\
2 & \stackrel{2}{?} \\
0 & ? \\
n & +1\end{array}$ & 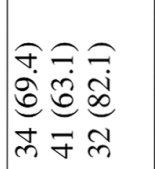 & 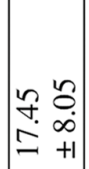 & 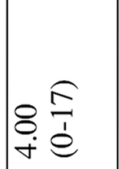 & 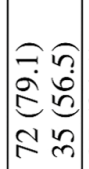 & 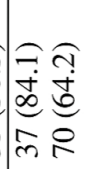 & 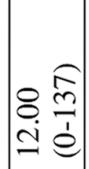 & $\mid \begin{array}{ll} & \widehat{n} \\
8 & 0 \\
8 & 0 \\
0 & 0 \\
0 & 0 \\
e\end{array}$ & 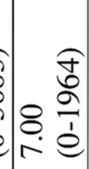 & 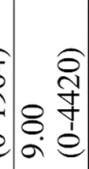 \\
\hline 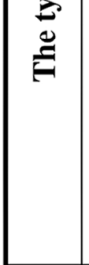 & 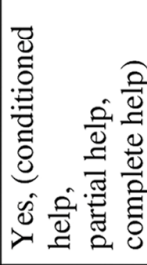 & 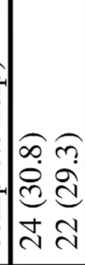 & 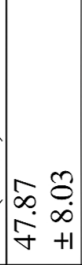 & 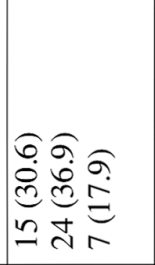 & 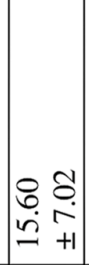 & ํㅠㅅ & 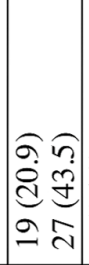 & 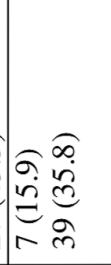 & 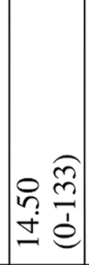 & $\begin{array}{ll}0 & 0 \\
8 & 0 \\
\dot{d} & 1 \\
& \vdots\end{array}$ & & $\hat{n}$ \\
\hline 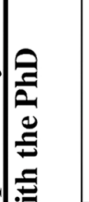 & $\stackrel{\vec{s}}{\stackrel{s}{s}}=$ & 总: & $\begin{array}{ll}2 & 0 \\
20 & 0 \\
0 & 0 \\
11 & 11\end{array}$ & 吾 & 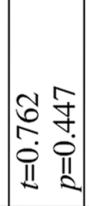 & 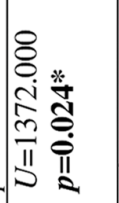 & 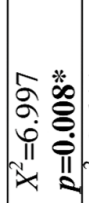 & 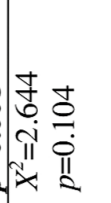 & 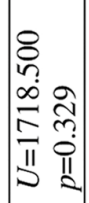 & 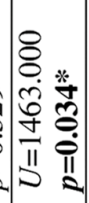 & 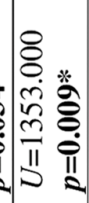 & 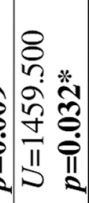 \\
\hline 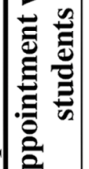 & z & 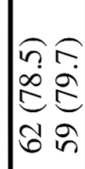 & \begin{tabular}{lr}
$m$ & $m$ \\
\hdashline & $\infty$ \\
0 & $\infty$ \\
$n$ & +1
\end{tabular} & 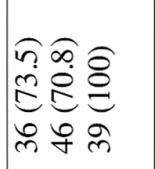 & \begin{tabular}{ll}
$n$ & $\infty$ \\
\hdashline & 0 \\
\hdashline & $\infty$ \\
$=$ & +1
\end{tabular} & & 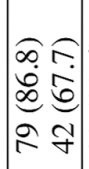 & 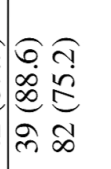 & 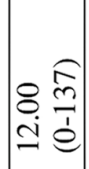 & 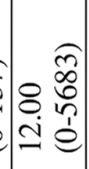 & 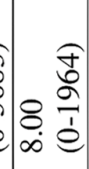 & : \\
\hline $\mid$ & 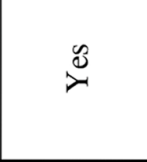 & 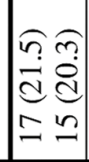 & 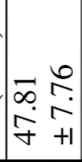 & 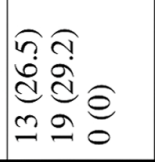 & 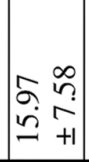 & 局 & 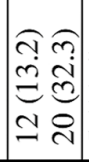 & 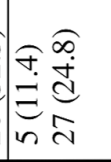 & 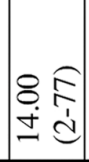 & & & 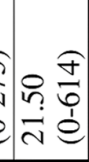 \\
\hline 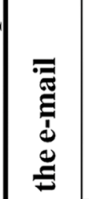 & $\stackrel{\overrightarrow{\mathrm{s}}}{\leftrightarrow}=$ & 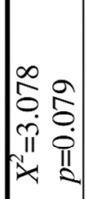 & 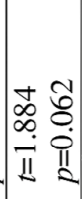 & 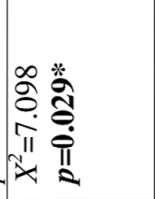 & 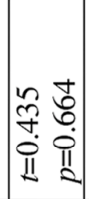 & 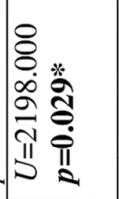 & 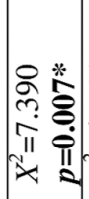 & 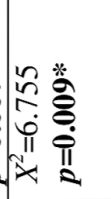 & 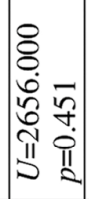 & 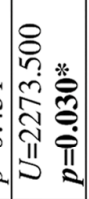 & 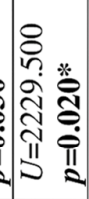 & 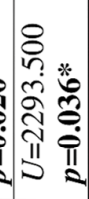 \\
\hline 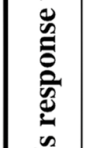 & ż & 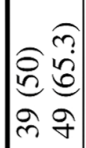 & $\mid$\begin{tabular}{cc}
0 & $\infty$ \\
\hdashline & $\stackrel{?}{?}$ \\
$i n$ & +1
\end{tabular} & 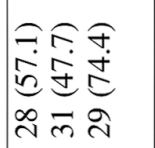 & 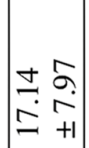 & $\underset{+}{\stackrel{E}{c}}$ & 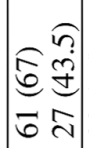 & 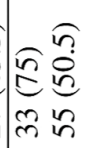 & 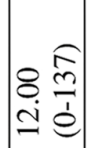 & 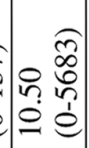 & 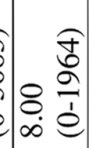 & 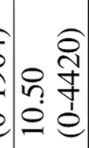 \\
\hline 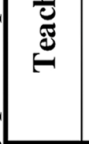 & $\overbrace{}^{\infty}$ & 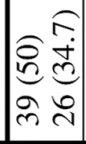 & 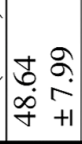 & 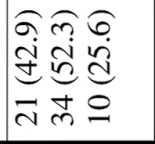 & 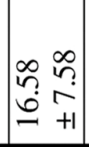 & ه & 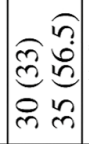 & 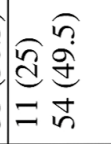 & $\begin{array}{ll}8 & \widehat{m} \\
\dot{ \pm} & \stackrel{1}{e}\end{array}$ & 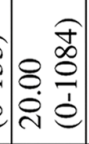 & $\mid \begin{array}{ll}8 & \widehat{0} \\
8 & 0 \\
\dot{1} & 1 \\
\pm & e\end{array}$ & 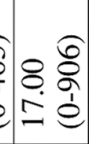 \\
\hline & \multirow[t]{2}{*}{$\begin{array}{l}\frac{y}{0} \\
\frac{\pi}{2} \\
\frac{\pi}{2} \\
\frac{\pi}{2}\end{array}$} & \multirow[t]{2}{*}{ 㫕 } & \multirow[t]{2}{*}{$\frac{a}{\pi}$} & \multirow{2}{*}{ 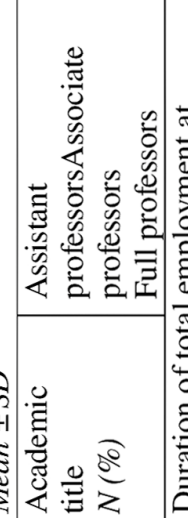 } & \multirow{2}{*}{ 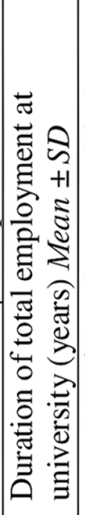 } & \multirow{2}{*}{ 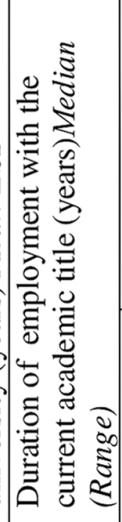 } & . & 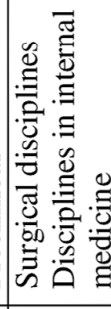 & \multirow{2}{*}{ 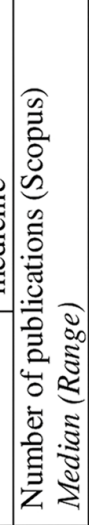 } & \multirow{2}{*}{ 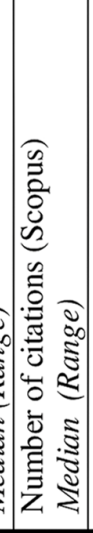 } & \multirow{2}{*}{ 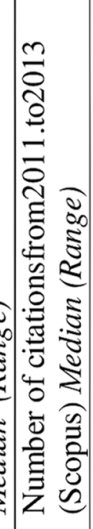 } & . \\
\hline & & & & & & & $\frac{8}{2}$ & $\frac{8}{2} \frac{2}{2}$ & & & & 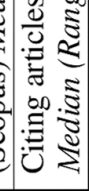 \\
\hline
\end{tabular}




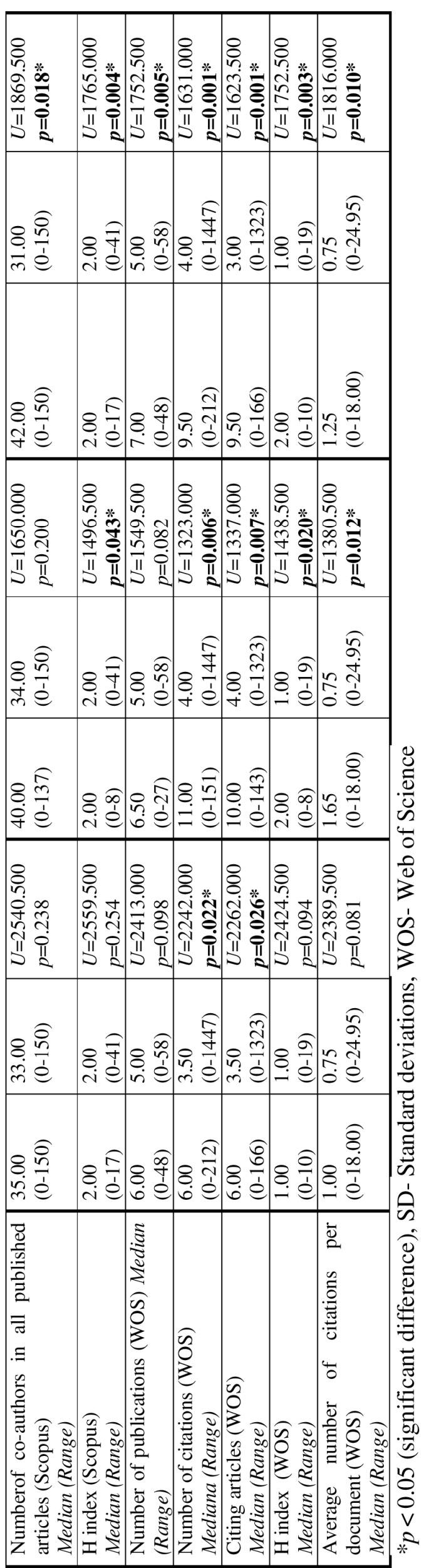


Table 2. Crude and adjusted odds ratios for the university teachers who replied to the e-mail vs. those who did not reply.

\begin{tabular}{||l|c|c|c|c|}
\hline \multicolumn{1}{|c|}{ Variables } & $\mathbf{p}$ & $\begin{array}{c}\text { Crude odds ratio } \\
\text { (95\% CI) }\end{array}$ & $\mathbf{p}$ & $\begin{array}{c}\text { Adjusted* odds ratio } \\
\text { (95\% CI) }\end{array}$ \\
\hline $\begin{array}{l}\text { Duration of employment with } \\
\text { the current academic title }\end{array}$ & 0.097 & $0.933(0.859-1.013)$ & $\mathbf{0 . 0 4 1 *}$ & $0.896(0.806-0.996)$ \\
\hline $\begin{array}{l}\text { Area (disciplines in internal } \\
\text { medicine or surgical } \\
\text { disciplines) }\end{array}$ & $\mathbf{0 . 0 0 7 *}$ & $2.946(1.352-6.418)$ & $\mathbf{0 . 0 2 6 *}$ & $2,846(1.135-7.139)$ \\
\hline $\begin{array}{l}\text { Area (preclinical or } \\
\text { clinic) }\end{array}$ & $\mathbf{0 . 0 0 4 *}$ & $2.636(1.355-5.128)$ & 0.357 & $1.473(0.646-3.357)$ \\
\hline \begin{tabular}{l} 
Number of publications (WOS) \\
\hline $\begin{array}{l}\text { Average number of citations } \\
\text { per document (WOS) }\end{array}$
\end{tabular} & 0.134 & $1.033(0.990-1.078)$ & $\mathbf{0 . 0 4 0 *}$ & $1.149(1.007-1.312)$ \\
\hline
\end{tabular}

Adjusted* for duration of employment with the current academic title, area (surgical disciplines or disciplines in internal medicine), area (preclinical or clinic), number of publications (Scopus), number of citations (Scopus), number of citationsfrom2011to2013 (Scopus), Citing articles (Scopus), Number of co-authors in all published articles (Scopus), H index (Scopus), Number of publications (WOS), Number of citations (WOS), Citing articles (WOS), H index (WOS) and Average number of citations per document (WOS). CI- Confidenc interval, $\mathrm{p}^{*}<$ 0.005 (significant difference), WOS- Web of Science.

${ }^{1}$ For the sake of clarity, values of ODDS ratios for variables without significant influence were omitted.

Table 3. Crude and adjusted odds ratios for the university teachers who made an appointment with the $\mathrm{PhD}$ students vs. those who did not.

\begin{tabular}{|l|c|c|c|c||}
\hline \multicolumn{1}{|c|}{ Variables } & $\mathbf{p}$ & $\begin{array}{c}\text { Crude odds ratio } \\
(\mathbf{9 5 \%} \text { CI) }\end{array}$ & $\mathbf{p}$ & $\begin{array}{c}\text { Adjusted odds ratio* } \\
\text { (95\% CI) }\end{array}$ \\
\hline $\begin{array}{l}\text { Duration of employment with } \\
\text { the current academic title }\end{array}$ & $\mathbf{0 . 0 1 1 *}$ & $0.836(0.727-0.960)$ & $\mathbf{0 . 0 1 6 *}$ & $0.782(0.641-0.955)$ \\
\hline $\begin{array}{l}\text { Area (preclinical or } \\
\text { clinic) }\end{array}$ & $\mathbf{0 . 0 0 6 *}$ & $3.135(1.398-7.030)$ & $\mathbf{0 . 0 3 7 *}$ & $3.194(1.074-9.498)$ \\
\hline
\end{tabular}

Adjusted* for sex, age (years), duration of employment with the current academic title, area (surgical disciplines or disciplines in internal medicine), area (preclinical or clinic), number of publications (Scopus), number of citations (Scopus), number of citationsfrom2011to2013 (Scopus), Citing articles (Scopus), Number of co-authors in all published articles (Scopus), H index (Scopus), Number of publications (WOS), Number of citations (WOS), Citing articles (WOS), $\mathrm{H}$ index (WOS) and Average number of citations per document (WOS). CI- Confidenc interval, $\mathrm{p}^{*}<0.005$ (significant difference), WOS- Web of Science.

${ }^{1}$ For the sake of clarity, values of ODDS ratios for variables without significant influence were omitted. 
Table 4. Crude and adjustedodds ratios for the university teachers who actually helped to the PhD student vs. those who did not

\begin{tabular}{|c|c|c|c|c|}
\hline Variables & $\mathbf{p}$ & $\begin{array}{l}\text { Crude odds ratio } \\
\quad(95 \% \mathrm{CI})\end{array}$ & $\mathbf{p}$ & $\begin{array}{l}\text { Adjusted odds ratio* } \\
\text { (95\% CI) }\end{array}$ \\
\hline Age (years) & $0.027 *$ & $0.948(0.905-0.994)$ & 0.616 & $1.019(0.947-1.097)$ \\
\hline $\begin{array}{l}\text { Duration of employment with } \\
\text { the current academic title }\end{array}$ & $0.015^{*}$ & $0.876(0.787-0.974)$ & 0.013* & $0.784(0.647-0.950)$ \\
\hline $\begin{array}{llll}\text { Area (disciplines } & \text { in } & \text { internal } \\
\text { medicine } & \text { or } & & \text { surgical } \\
\text { disciplines) } & & & \end{array}$ & 0.018* & $2.945(1.200-7.227)$ & 0.147 & $2.409(0.734-7.901)$ \\
\hline $\begin{array}{l}\text { Area (preclinical or } \\
\text { clinic) }\end{array}$ & $0.003 *$ & $2.923(1.434-5.960)$ & 0.353 & $1.608(0.591-4.376)$ \\
\hline Number of publications (WOS) & $0.020 *$ & $1.056(1.009-1.106)$ & $0.022 *$ & $1.192(1.026-1.386)$ \\
\hline $\begin{array}{l}\text { Average number of citations } \\
\text { per document (WOS) }\end{array}$ & $0.036^{*}$ & $1.131(1.008-1,270)$ & 0.058 & $1.506(0.986-2.300)$ \\
\hline
\end{tabular}

Adjusted* for sex, age (years), duration of employment with the current academic title, area (surgical disciplines or disciplines in internal medicine), area (preclinical or clinic), number of publications (Scopus), number of citations (Scopus), number of citationsfrom2011to2013 (Scopus), Citing articles (Scopus), Numberof coauthors in all published articles (Scopus), H index (Scopus), Number of publications (WOS), Number of citations (WOS), Citing articles (WOS), H index (WOS) and Average number of citations per document (WOS). CI- Confidenc interval, $\mathrm{p}^{*}<0.005$ (significant difference), WOS- Web of Science.

${ }^{1}$ For the sake of clarity, values of ODDS ratios for variables without significant influence were omitted.

\section{DISCUSSION}

Our study revealed influence of a few factors on willingness to offer help among the faculty, and most of them were associated with research activities and results. While sex of the facultywas not important factor, the results showed that younger professors were more ready to help to $\mathrm{PhD}$ students (Table 1). Only a handful of Full Professors responded to the e-mail, compared to the Associate Professors and Assistant Professors. Not a single Full Professor made an appointment with the $\mathrm{PhD}$ students (Table 1), and they responded to the e-mail much less frequently than Associate and Assistant Professors. Faculty who had more recently been employed at the current academic position were more ready to interact with the PhD students (Tables 1, 2, 3 and 4). Another study showed low total response rate to undergraduate students' requests for additional explanations, but on the contrary to our results, younger teachers were less will- ing to help, possibly because of high burden of overall university duties ${ }^{12}$.

An interesting finding in our study was that faculties who are teaching students in preclinical disciplines were more ready to help than professors of clinical disciplines. It was found that professors of preclinical disciplines were 2.5 times more likely to respond to e-mails, over 3 times more likely to schedule appointments and about 3 times more likely to provide some form of help to the $\mathrm{PhD}$ students, compared to the professors who are teaching clinical disciplines. In the same time, professors of preclinical disciplines had better research performance with higher publication and citation scores. Although in clinical disciplines, higher academic grade is usually associated with higher research performance and publication rate $^{17}$; in our study it was the opposite, since faculty from preclinical disciplines, who were having lower academic positions, had higher publication and citation scores than the faculties from clinical disciplines. Therefore, 
higher research experience of preclinical faculties was probably responsible for greater willingness to help to the PhD students with methodological advice.

Faculties of internal diseases disciplines in our study were about 3 times more likely to respond to e-mail of the $\mathrm{PhD}$ students and to provide assistance, compared with professors of surgical disciplines. Other studies also showed lower involvement rate of professors of surgical disciplines in teaching activities $^{18}$, which could partly be explained by their high burden of clinical work and more stressful working conditions.

Actually, the strongest predictors of high willingness to help and transfer knowledge in our study were publication and citation scores, including $\mathrm{H}$ index calculated from both Scopus and WOS databases. Although average value of $\mathrm{H}$ index for our study participants was low (H index from 5 to 9- well established publication record; from 10 to 14 excellent publication record; $\mathrm{H}$ index over 15- exceptional publication record $)^{19}$, it still determined their attitude toward the students, showing that in a way it is linked also with personal ethics.

The study participants with greater number of co-authors in all their published articles (Scopus) helped the PhD students more frequently, compared with a group of professors with smaller number of co-authors (Table 1). It seems that ability to work in a team and organize people is also associated with higher personal ethical standards: it was shown that positive attitude towards the teamwork was linked with higher knowledge of medical ethics ${ }^{20}$.

One possible limitation of our study was that the outcome of the request for help could depend on how a PhD student presented or explained what kind of help he or she needed, both within the e-mail correspondence and while meeting a professor. For example, some of the professors ended the conversation after finding out that assistance from their specialized field of work was not required, although the request was centered on basic research methodology, common to all fields.
Our study showed that responsible behavior of faculty in regard to knowledge transfer to $\mathrm{PhD}$ students is associated with younger age, shorter employment at university and with higher publication and citation scores. It seems that an advanced research environment and higher academic achievements positively influence development of awareness about teaching responsibilities among the faculty. Universities should adopt and publicize a code of conduct of researchers which clarifies expectations of helping students, which then should be publicly available.

Awareness of other, less frequent forms of misbehavior, should also be investigated. This will require innovative research methods, like simulation of roles of $\mathrm{PhD}$ students or other kinds of researchers. The results of this study could be used in the process of teaching ethics at $\mathrm{PhD}$ studies, as a case study. This would help to make $\mathrm{PhD}$ students more aware of their rights.

\section{ACKNOWLEDGEMENTS}

This work was supported by the Serbian Ministry of Education under Grant 175007, and by Ministry of Science, Montenegro, under Grant No 404.

\section{REFERENCES}

1. Vähäkangas K. Research ethics in the post-genomic era. Environ Mol Mutagen 2013; 54(7): 599-610.

2. Gilbert FJ, Denison AR. Research misconduct. ClinRadiol 2003; 58(7): 499-504.

3. Munby J, Weetman DF.Joint Consensus Conference on Misconduct in Biomedical Research. Indoor Built Environ 1999;8: 336-8.

4. Howe KR, Michelle SM. Ethics in educational research. Rev ResEduc1999; 24(1): 21-60.

5. Gardner H. The ethical mind. A conversation with psychologist Howard Gardner. HarvardBus Rev 2007; 85(3): 51-6.

6. MacDougall M, Riley SC. Initiating undergraduate medical students into communities of research practice: what do supervisors recommend? BMC Med Educ 2010; 10: 83.

7. Barondess JA. A brief history of mentoring. Trans Am ClinClimatolAssoc 1995; 106: 1-24.

8. Lev EL, Kolassa J, Bakken LL. Faculty mentors' and students' perceptions of students' research self-efficacy. Nurse Educ Today2010; 30(2): 169-74. 
9. Mitchell T, Carroll J. Academic and research misconduct in the PhD: issues for students and supervisors. Nurse Educ Today 2008; 28(2): 218-26.

10. Woods SK, Burgess L, Kaminetzky C, McNeill D, Pinheiro S, Heflin MT. Defining the roles of advisors and mentors in postgraduate medical education: faculty perceptions, roles, responsibilities, and resource needs. J Grad Med Educ2010; 2(2): 195-200.

11. Sambunjak D, Straus SE,Marusić A. Mentoring in academic medicine: a systematic review. JAMA 2006; 296(9): 1103-15.

12. Masić I, Dedović-Halilbegović G, Novo A,Izetbegovíc S. Quality assesment of education at Faculty of Medicine University of Sarajevo. Med Arh2006; 60(6): 396400.

13. Hirsch JE. An index to quantify an individual's scientific research output. ProcNatlAcadSci U S A 2005; 102(46): 16569-72.

14. Elsevier, B.V. Scopus. Retrieved December 1, 2013, from http://www.scopus.com

15. Thomson, R. ISI Web of Knowledge. Retrieved December 1, 2013, from http://www.isiwebofknowledge.com
16. Forggin, S. The H index and Journal impact Factors. Retrieved December 15,2013, from http://www.library. auckland.ac.nz/subject-guides/med/pdfs/H-index\%20and\%20impact\%20factors.pdf

17. Hurley RW, Zhao K, Tighe PJ, Ko PS, Pronovost PJ, $\mathrm{Wu}$ CL.Examination of publications from academic anesthesiology faculty in the United States. AnesthAnalg 2014; 118(1): 192-9.

18. Shimura T, Aramaki T, Shimizu K, Miyashita T, Adachi K,Teramoto A. Implementation of integrated medical curriculum in Japanese medical schools. J Nippon Med Sch2004; 71(1): 11-6.

19. De Groote SL, Raszewski R.Coverage of Google Scholar, Scopus, and Web of Science: A case study of the hindex in nursing. Nurs Outlook 2012; 60 (6): 391-400.

20. Fang JT, Ko YS, Chien CC, Yu KH.Assessing medical students' performance incore competencies using multiple admission programs for colleges anduniversities: from the perspective of multi-source feedback. Biomed J 2013; 36(4): 188-9. 